\title{
Endoscopic retrograde cholangiopancreatography in the elderly: results of a retrospective study and a geriatricians' point of view
}

Marianna Galeazzi ${ }^{1}$, Paolo Mazzola ${ }^{1,2^{*}}$, Breanna Valcarcel ${ }^{5}$, Giuseppe Bellelli ${ }^{1,2,3}$, Marco Dinelli ${ }^{4}$, Giulio Maria Pasinetti ${ }^{5,6}$ and Giorgio Annoni $i^{1,2,3}$

\begin{abstract}
Background: The incidence of biliary tract pathology is growing with an age-related trend, and progresses as the population ages. Endoscopic Retrograde Cholangiopancreatography (ERCP) represents the gold standard for treatment in these cases, but evidence about its safety in the elderly is still debated.

Methods: We retrospectively analyzed the clinical records of all patients aged $\geq 65$ undergoing ERCP between July 2013 and July 2015. Of 387 ERCP cases, 363 ( 94\%) were completed entirely. The mean age of the study population ( $n=363$ ) was 79.9 years old (range 70-95), with 190 subjects aged 70-79 and 173 older than 80. We recorded demographics, Charlson Comorbidity index (CCI), American Society of Anesthesiologists (ASA) physical status classification score, indication for the use of the ERCP procedure, and clinical outcomes. Then, we tested all variables to identify the potential risk factors for complications associated with the procedure.

Results: The older group (those $\geq 80$ years old) showed significantly more patients with ASA Classes III-IV than the younger one (those $\leq 79$ years old). Interestingly, the CCI was higher in the younger group $(p=0.009)$. The overall complication rate was $17.3 \%$ without inter-group differences. Older age, sex, CCl and intra-ERCP procedures were not related to a higher risk of complications, and the multivariate regression did not identify any of the considered variables to be an independent risk factor for complications.
\end{abstract}

Conclusion: ERCP appears as safe in the patients aged 80 years and older, as it is in those aged 70-79 years old in our study, however, a selection bias may affect these findings. A study including a comprehensive geriatric assessment will contribute to shedding light on this issue.

Keywords: Endoscopic retrograde cholangiopancreatography, ERCP, Endoscopy, Elderly, Procedural complications

\section{Background}

The incidence of biliary tract pathology is growing due to the overall aging of the population worldwide, and this is particularly evident among the number of those over the age of $80^{1}$. Endoscopic Retrograde Cholangiopancreatography (ERCP) represents the gold standard exploratory technique for the treatment of biliary or

\footnotetext{
*Correspondence: paolo.mazzola@unimib.it

'University of Milano-Bicocca, School of Medicine and Surgery, U8 Building,

Floor 4, Lab 4045, Via Cadore, 48 - 20900, Monza, MB, Italy

${ }^{2}$ NeuroMl - Milan Center for Neuroscience, Clinical Neurosciences research area, Milan, MI, Italy

Full list of author information is available at the end of the article
}

pancreatic tract pathology, and it could be often performed with therapeutic intent by realizing procedures such as insertion of bile duct stents and/or endoscopic sphincterotomy. However, evidence about its safety in the elderly is still controversial.[1-5] A recent systematic review by Day et al.[4] explored the issue of effectiveness and safety of ERCP in octogenarians (i.e. $\geq 80$ years old) versus younger seniors and late adults, showing that perforation, hemorrhage, biliary infection, and other main complications of the procedure were not statistically different among the three groups. However, despite the sample size of the meta-analysis, patients did not 
undergo a comprehensive approach to be characterized. Instead of more inclusive tools, the frequency of symptoms, the proportion of some comorbid conditions (namely hypertension, diabetes, coronary heart and cerebrovascular diseases, and "others"), and the American Society of Anesthesiologists (ASA) physical status classification score were the parameters collected.[6] From a geriatric perspective, the assessment of older patients is an interdisciplinary process that should include multiple domains, such as functional and nutritional status, cognition, accurate evaluation of comorbidity, and many others[7].

In order to add knowledge to this scenario and to understand if this gap in patient's assessment applies to our population, we retrospectively analyzed the clinical records of older patients undergoing ERCP in the endoscopy unit of an Italian university hospital. Our aim was to assess how the specialists in endoscopy evaluate patients undergoing ERCP, the success rate of this procedure, and the incidence of complications in octogenarians compared to that of younger seniors (aged $<80$ years old).

\section{Methods}

\section{Study design and subjects}

This retrospective observational single-center study was carried out at San Gerardo Hospital ASST Monza, a large Italian university hospital. We retrieved data from the Endoscopy Unit electronic registry, collecting all the ERCP procedures that took place from July 1st, 2013 to July 31st, 2015 in an electronic spreadsheet. We considered only ERCPs performed in patients ages 65 and older, and divided the study population into two groups according to the age criterion (65-79 vs. $\geq 80$ ).

All data were de-identified according to the Italian legislation "Data Protection Code - Legislative Decree no. 196/2003" (available at http://www.garanteprivacy.it/ home_en/italian-legislation) for the protection of personal information. Permission for the use of this information was granted by a specific consent form signed by each patient or his/her legal representative for undergoing the endoscopy procedure. The informed consent forms are stored in the archive of San Gerardo Hospital ASST Monza (Monza, Italy).

For every ERCP procedure performed, we collected the following information: patient's age and gender, comorbid conditions, date, indication and duration of the procedure, ASA physical status classification score, [6] duration of sedation/anesthesia, additional procedures, complications, success in addressing the indications, diagnosis, and whether the ERCP was completed or not. Multiple procedures performed in the same patient were also taken into account.

With more detail, the ASA score is a qualitative system originally developed to describe the pre-operative fitness of patients scheduled for surgical procedures. It currently consists of 6 classes, from ASA 1 (normal healthy subject) to ASA 6 (a declared brain-dead patient). The higher the ASA class, the poorer the patient's health status is considered to be. Comorbid conditions were assessed with the Charlson Comorbidity Index (CCI), [8] a weighted index that assigns a different risk score (from 1 to 6) to 22 conditions and their related relative risk of 1-year mortality. The higher the CCI score, the higher the risk of 1-year mortality.

\section{Procedures}

All the ERCP procedures were performed by four experienced gastroenterologists with 10 or more years of expertise in endoscopy and in this procedure specifically.

Patients underwent sedation as indicated in our Endoscopy Unit sedation protocol, which agrees with national guidelines, available at: http://www.sied.it/files/ 12ConigliaroFantiGIED3_14.pdf; http://www.sied.it/files/ Lineeguidaperlasedazioneinendoscopiadigestiva.pdf .

It should be considered that regulations limit the drugs that non-anesthetists could administer to meperidine or fentanest and midazolam. In ASA I and II patients who do not require a urgent procedure, the endoscopic team performed moderate sedation with opioids and midazolam. Sedation is constantly monitored as it is used for other endoscopic procedures.

ASA III/IV patients and those who need to undergo urgent ERCP were sedated and monitored by an anesthetist who decided to perform deep sedation or general anaesthesia according to its judgement and the subject's clinical conditions at the moment of undergoing the procedure. All urgent ERCPs were performed in the operating room irrespectively of the type of ERCP and sedation chosen.

\section{Definition of complications}

The adverse events we recorded during and/or in response to ERCP procedures were defined as follows:

- Pancreatitis: the onset of new abdominal pain with at least a three-fold elevation of serum amylase or lipase levels, at least $24 \mathrm{~h}$ after the procedure;

- Perforation: retroperitoneal or bowel-wall perforation, as evidenced by imaging techniques;

- Bleeding: clinical evidence of hemorrhage, with a decrease in hemoglobin $>2 \mathrm{~g} / \mathrm{dL}$ or the need for endoscopic or transfusion treatment;

- Cholangitis: temperature of more than $38{ }^{\circ} \mathrm{C}$ for $24-$ $48 \mathrm{~h}$ occurring after the procedure and thought to have a biliary cause, without evidence of concomitant infection;

- Cardiopulmonary adverse events: myocardial infarction, cerebrovascular accident, congestive heart failure, cardiac/respiratory arrest, arrhythmia, hypoxemia 
(oxygen saturation $<90 \%$ ), hypotension (systolic blood pressure $<90 \mathrm{mmHg}$ ), bradycardia (heart rate $<60$ beats/ $\mathrm{min}$ ), tachycardia (heart rate $>110$ beats/min), or vasovagal response, all of which were attributed to the performance of an ERCP procedure;

- Mortality related/not related to the performance of the ERCP: death within $48 \mathrm{~h}$ after a procedure with complications or incomplete endoscopic treatment (for example, relief of bile duct obstruction was not achieved by the intervention) was regarded as a procedure-related death.

These complications were defined by Cotton's criteria [9].

\section{Outcomes}

After describing the parameters and information we were able to collect from the hospital registry, the primary outcome was to assess the incidence of complications or deaths associated with ERCPs.

The secondary outcome was the identification of the baseline characteristics and ERCP-related variables able to predict the onset of complications.

\section{Statistical analysis}

All analyses were performed with the software SPSS Version 23.0 (IBM Corp., Armonk, NY, USA). Continuous variables were expressed as mean \pm standard deviation (SD). Categorical variables were expressed as frequencies and percentages. Comparisons between the study groups were performed with the Student's t-test for continuous variables and the Chi-square test for categorical variables. All the comparisons were two-tailed.

The analyses of the risk factors for ERCP-related complications were performed through univariate and multivariate logistic regression models. Significance was obtained for values of $p<0.05$.

\section{Results}

\section{Patients' characteristics}

Seven hundred and thirty-two ERCPs were performed at San Gerardo Hospital from July 1st 2013 to July 31st 2015. Of them, 485 (66.3\%) were performed in patients ages $65-95$. To date, $9 \%$ (33 subjects) of the population was nonagenarian (i.e. $\geq 90$ years old). Four hundred and sixty-five patients were hospitalized at San Gerardo Hospital, while 20 subjects were admitted into other nearby hospitals but underwent ERCP in our Endoscopy unit; 53 ERCPs were considered as urgent procedures.

Ninety-seven patients underwent more than one procedure for technical reasons (e.g.: stent placement and removal) or for treatment of a second recurrence of a common bile duct (CBD) stone. Specifically, one patient underwent seven ERCPs, three patients underwent five ERPCs, eight patients underwent four ERCPs, 34 underwent three procedures, and 51 underwent ERCP twice.

Interestingly, the CCI was significantly higher in the younger group compared to that of the older group (Table 1). However, considering the age-adjusted CCI, we observed the opposite situation with the CCI being higher in the older group than that of the younger one, but this difference did not reach statistical significance.

The ASA scores, considering each separate class, presented significant differences between the groups ( $p=$ 0.048 ). We also grouped the ASA scores between classes I-II (low intra-operative risk) and classes III-IV (moderate-to-high intra-operative risk), rather than all four classes individually, and we observed that the older group had

Table 1 Baseline characteristics of the enrolled patients

\begin{tabular}{|c|c|c|c|c|}
\hline Groups & $\begin{array}{l}\text { Age } 65-79 \\
N=280\end{array}$ & $\begin{array}{l}\text { Age } \geq 80 \\
N=185\end{array}$ & $\begin{array}{l}\text { All } \\
n=465\end{array}$ & $\overline{p \text {-value }}$ \\
\hline Mean age, years & $72.78 \pm 4.4$ & $85.29 \pm 4.18$ & $77.6 \pm 7.64$ & $<0.001$ \\
\hline Gender, n (\%) & & & & $<0.001$ \\
\hline Male & $146(52.1)$ & $66(35.7)$ & 212 & \\
\hline Female & $134(47.9)$ & $119(64.3)$ & 253 & \\
\hline Charlson Comorbidity Index (CCI), mean & $1.6 \pm 2.0$ & $1.2 \pm 1.4$ & $1.2 \pm 1.8$ & 0.008 \\
\hline Age-adjusted CCl, mean & $4.4 \pm 2.0$ & $5.3 \pm 7.0$ & $4.8 \pm 1.8$ & 0.059 \\
\hline ASA score, $n$ & & & & 0.048 \\
\hline । & $2(0.7)$ & - & 2 & \\
\hline$\|$ & $210(75.0)$ & $113(61.1)$ & 323 & \\
\hline III & $63(22.5)$ & $69(37.3)$ & 132 & \\
\hline IV & $5(1.8)$ & $3(1.6)$ & 8 & \\
\hline V & - & - & - & \\
\hline Urgent ERCP, n (\%) & $27(9.6)$ & $26(14.1)$ & $53(11.6)$ & 0.179 \\
\hline
\end{tabular}

Data are presented as mean $\pm \mathrm{SD}$ or as a number and percentage. ASA American Society of Anesthesiologists 
a significantly greater proportion of class III-IV ASA scores $(38.9 \%$ vs. $26.7 \%$ of the younger group, $p=0.011)$.

Table 2 shows that the main indication for ERCP intervention was CBD stones, followed by stent placement/ removal. No statistical difference between the groups was found.

\section{ERCP: Procedures, success rate and complications}

Among a total of 465 procedures, 438 were completed: 265 ERCPs belonged to the younger group, 173 to the older one. The rates of success of ERCP in the younger and older group were $94.6 \%$ and $93.7 \%$, respectively.

The 27 failures were due to complications (3/27: 2 bradycardia and 1 perforation), single diverticulum associated ampulla (17/27), altered surgical anatomy (3/27), and multiple large diverticula with no identification of the Vater ampulla (4/27).

All procedures performed during the ERCP and the diagnoses are presented in Table 3 and Table 4, respectively. No statistical differences between the groups were found. CBD stones were the most frequent diagnosis.

Fifty-three complications were related to the procedure (Table 5). In the younger group, the complication rate was $13.2 \%$ vs. $10.0 \%$ in the older one.

All complications were higher in the younger group. Two out of 4 patients who had bleeding (younger group) used to take aspirin. To date, no one used oral anticoagulants.

Seven complications (13\%) happened during urgent ERCPs, six in the younger group (four sepsis, two perforations), and one in the older group (sepsis). No statistical difference between these groups emerged.

Ten episodes were considered as not related to ERCP: fever without sepsis (eight patients), ischemic stroke $(n=1)$, and diarrhea $(\mathrm{n}=1)$.

Nine patients died after the ERCP procedure, three of whom due to ERCP-related shock. Those cases underwent ERCP for CBD stones and for stent removal, and presented only cardiovascular comorbidities.

Six patients, of the nine who died, passed because of shock from septic cholangitis, despite successful

Table 2 Indications for ERCP procedures in the study groups and in the population as a whole

\begin{tabular}{llll}
\hline Indication & Age 65-79 & Age $\geq 80$ & All \\
& $\mathrm{N}=280$ & $\mathrm{~N}=185$ & $\mathrm{~N}=465$ \\
\hline CBD stones/ biliary colic & $92(32.9)$ & $74(40.0)$ & $166(35.7)$ \\
Stenosis & $51(18.2)$ & $28(15.1)$ & $79(17.0)$ \\
Acute Pancreatitis & $16(5.7)$ & $12(6.5)$ & $28(6.0)$ \\
Stent placement or removal & $66(23.5)$ & $40(21.6)$ & $106(22.8)$ \\
Cholangitis & $40(14.3)$ & $29(15.7)$ & $69(14.8)$ \\
Other & $15(5.4)$ & $2(1.1)$ & $17(3.7)$ \\
\hline
\end{tabular}

All indications are reported as a number and percentage (\%) completion of the ERCP. Their ages were $68,69,79,89$, 92, 93; three of these ERCPs were urgent procedures (performed in the patients aged 79, 89, and 92 years old).

In order to test the single variables in their potential association to the onset of complications, we performed univariate logistic regression analyses, but we did not find significant relationships with the outcome (Table 6). Even the type of invasive procedure performed during ERCP was not related with experiencing complications. In particular, unsuccessful procedure (OR 1.45, $p=$ 0.437), sphincterotomy (OR 1.14, $p=0.630$ ), stenting (OR $0.62, p=0.078$ ), and the combination of sphincterotomy+stenting (OR 1.31, $p=0.303$ ) did not relate to the outcome (Table 6).

We also performed a multivariate logistic regression analysis including age (groups of those $<80$ vs. those $\geq 80$ years), gender, CCI (groups of those with scores of $<4$ vs. those with scores of $\geq 4$ ), type of intervention (categorical), and urgency of the procedure. Not surprisingly, age, gender, type of procedure and urgency did not result as significantly associated with complications. However, the dichotomized CCI risk scores (in $<4$ versus $\geq 4$ ) resulted as independently and significantly correlated with the outcome (Odds Ratio, OR $=0.29$, 95\% Confidence Interval, C.I.: $0.09-0.99, p=0.049$ ).

\section{Discussion}

The demand for ERCP is increasing in the elderly and it is important to know the procedure risks and benefits in older patients. The two study groups were different in terms of age, sex, CCI and ASA score.

The older group (ages 80 and above) had a significantly higher number of female patients and this is probably due to the different life expectancy between men and women. This data was recently confirmed in the literature [10].

The older group showed a lower mean CCI, thus raising a question about the existence of a possible selection bias. However, if we consider the age-adjusted CCI, the mean values were significantly higher in older patients. In order to perform ERCP procedures, these data suggest that gastroenterologists specializing in endoscopy probably rely on inclusion criteria that do not take into account the multi-faceted characteristics of the geriatric patient. However, the CCI - age-adjusted or not should not be considered as a stand-alone assessment tool. Those inclusion criteria often imply that older subjects are selected for their apparent "fitness" according to comorbidity, which is a crucial limitation. Even the ASA physical status classification system - despite being validated for assessing fitness of patients undergoing surgery - cannot be considered as a stand-alone tool for the evaluation of elderly subjects scheduled for ERCP. Not 
Table 3 Intra-ERCP procedures in the study groups and in the population as a whole. All procedures are reported as a number and percentage (\%)

\begin{tabular}{llll}
\hline Procedures & Age 65-79 & Age $\geq 80$ & All \\
& $N=280$ & $N=185$ & N=465 \\
\hline Sphincterotomy, n \% & $74(28)$ & $47(27)$ & $121(27.6)$ \\
Stent placement or removal, n \% & $101(38)$ & $65(37.5)$ & $166(37.9)$ \\
Sphincterotomy and stent placement, n \% & $85(32)$ & $59(34)$ & $144(32.8)$ \\
Other, $\mathrm{n} \%$ & $5(2)$ & $2(1.5)$ & $7(1.7)$ \\
Incompleted procedure & $15(5.3)$ & $12(6.4)$ & $27(5.8)$ \\
\hline
\end{tabular}

surprisingly, ASA score is mainly a qualitative rather than a quantitative index, theoretically insufficient to describe the complexity of the geriatric population.

A further possible signal of selection bias is the fact that ASA III-IV patients were significantly prevalent in the older group, in potential contrast with the simultaneous presence of a lower index of comorbidity in this age group. Again, even by dichotomizing the CCI in $<4$ versus $\geq 4$, the younger group displays a higher number of patients with $\mathrm{CCI} \geq 4$ than the older one, confirming that patients scheduled for ERCP undergo uneven selection criteria. Those criteria tend to exclude the more comorbid elderly subjects from the procedure, presumably according to the assumption that higher comorbidity translates into higher incidence of complications and mortality related to this procedure.

We agree that several older patients are able to tolerate ERCP, and that older people with comorbidities are at higher risk for some complications compared to those without comorbid conditions,[11] but understanding the "big picture" goes beyond the mere collection of medical history or fitness status. When facing older individuals, a comprehensive geriatric assessment (CGA) should be considered as the preferable way to assess their health status in its entirety. The CGA considers the multiple aspects of the elderly, namely comorbid conditions, polypharmacy, functional level, cognition, and nutritional status among other factors which once combined provide a more reliable prognostic estimation than the ASA score or a comorbidity index alone. We are confident that the role of a CGA will be increasingly relevant for risk stratification and risk management of these patients, or at the very least, a useful guide for avoiding ERCP in subjects who have a high risk of experiencing more harm than benefits from the procedure.

Back to our findings in this study, they were consistent with the literature in terms of indications, diagnoses, $[1,10]$ and success rates $(92.8 \%$ vs. $93.5 \%$, for the younger and older group, respectively) [12-15]. The frequencies of ERCP-related complications were $13.2 \%$ in the young group and $10.0 \%$ in the older, which is slightly higher than the rates reported in the literature (2.5\% to $12 \%)$. ERCP-related death incidence was $0.7 \%$ and $0.5 \%$ respectively, in line with previous reports, [12-25] though the only systematic review of ERCPs in the elderly suggests higher rates of death in octogenarians and nonagenarians than in younger patients.[4] We also observed that advancing age was not related to complications, neither in the case of the continuous variable, nor the dichotomized one $(\mathrm{OR}=0.99$, 95\% C.I.: 0.95-1.02, $p=0.399$; and OR 0.67, 95\% C.I. 0.40$1.12, p=0.128$, respectively). This is in line with our hypothesis, i.e. that age per se does not carry more complications. However, the finding should still be interpreted carefully because of several limitations.

Neither the indications (given a priori) nor the diagnoses (given ex-post, during or after ERCP completion) resulted as significantly related to complications, and again this could be due to the above-mentioned

Table 4 ERCP diagnoses in the study groups and in the whole population. All diagnoses are. reported as number of patients and percentage (\%)

\begin{tabular}{llll}
\hline Diagnosis & Age 65-79 & Age $\geq 80$ & All \\
& $N=265$ & $N=173$ & N=438 \\
\hline CBD stones & $119(44.9)$ & $87(50.3)$ & $81(18.5)$ \\
Stenosis & $50(18.9)$ & $31(17.9)$ & $71(16.2 \%)$ \\
$\quad$ Malignant stenosis & $40(15.1 \%)$ & $31(17.9 \%)$ & $122(27.9)$ \\
$\quad$ Inflammatory stenosis & $10(3.8 \%)$ & 0 & $28(6.4)$ \\
Stent placement or removal & $73(27.6)$ & $49(28.3)$ & $6(3.5)$ \\
Other & $22(8.3)$ & & 2 \\
\hline
\end{tabular}

CBD common bile duct 
Table 5 ERCP-related complications

\begin{tabular}{llll}
\hline Complications & Age 65-79 & Age $\geq 80$ & All \\
\hline Cholangitis-Septic cholangitis & 17 & 6 & 23 \\
Cardiopulmonary & 6 & 3 & 9 \\
Bleeding & 4 & 0 & 4 \\
Acute Pancreatitis & 5 & 4 & 9 \\
Perforation & 3 & 2 & 5 \\
Death ERCP related & 2 & 1 & 3 \\
Total ERCP-Related complications & $\mathbf{3 7}$ & $\mathbf{1 6}$ & $\mathbf{5 3}$ \\
\hline
\end{tabular}

selection bias. Unexpectedly, the type of invasive procedure performed during ERCP was not related with experiencing complications (Table 6).

Pertaining to the multivariate analysis, it should be noted that the sign of the relationship between CCI (dichotomized between $<4$ vs. $\geq 4$ ) and complications is negative, thus it seems that the higher the CCI class, the lower the risk of complications. This fact does not agree with the literature, but it should be interpreted. Indeed, Table 1 showed that a higher number of patients with $\mathrm{CCI} \geq 4$ belong to the 70-79 age group, and that they do not experience more complications than the older group, opening various interpretations but also confirming the potential impact of the selection bias for this procedure. However, the literature findings do not agree in terms of risk factors for the onset of complications [12-16].

The first limitation of our study is represented by the relatively limited sample size $(n=363)$, though this is similar to that of other studies $[3,23,26]$. The size issue probably explains also part of the difference between findings in the literature. This also gives a possible reason for the absence of significant associations observed at the univariate and multivariate analyses. On the one hand, this is quite unexpected because some of them should be theoretically correlated with complications, e.g. the degree of invasiveness of the procedure (performing sphincterotomy+stent placement is different than an ERCP executed for diagnostic purposes only). On the other hand, those results could be due to the selection bias for this procedure, which is expected to alter the normal correlations, especially if the study population is somewhat limited in size.

In addition, due to the single-centered and retrospective design, our findings cannot be generalized to other acute care settings. Furthermore, we retrieved hospital records but we do not have information about the post-discharge phases of care of the studied ERCP-treated patients. Finally, a major limitation is the lack of a structured, homogenous assessment of the ERCP candidates pre-operatively, which translates to the selection bias enlightened by our analysis. The only study that tried to assess the ERCP-related risk in the elderly population considering multiple domains suggested to include the Duke Activity Status Index (DASI, ranging from 0 to 58.2) to evaluate the functional capacity of subjects with cardiovascular disorders, with higher scores indicating better functional status. Further studies are necessary to confirm the importance of multidimensional scores, i.e. one or more tools able to describe the older patient comprehensively [26].

Given these limitations, this study provides insight and paves the path for including more comprehensive assessment tools in future prospective investigations, which are strongly needed. The final objective is to better characterize the elderly patients pre-operatively and select candidates who will have the highest probability of benefiting from ERCP, reducing the risk of developing intra- and post-operative complications.

\section{Conclusions}

ERCP remains a procedure burdened by many known risks, especially if performed with urgency and in the

Table 6 Univariate logistic regression analysis of the potential predictors for the onset of ERCP-related complications

\begin{tabular}{llll}
\hline Potential risk factors & Odds Ratio & $95 \%$ Confidence Interval & $p$-value \\
\hline Age (continuous) & 0.99 & $0.95-1.02$ & 0.399 \\
Age group ( $\geq 80)$ & 0.67 & $0.40-1.12$ & 0.128 \\
Sex, female & 0.81 & $0.50-1.32$ & 0.390 \\
Charlson Comorbidity Index (CCl, continuous) & 0.96 & $0.83-1.10$ & 0.547 \\
Age-adjusted CCI (continuous) & 0.93 & $0.81-1.06$ & 0.260 \\
ASA score (continuous) & 1.03 & $0.64-1.66$ & 0.901 \\
ASA group (III-IV) & 1.04 & $0.59-1.85$ & 0.886 \\
Procedure not completed & 1.45 & $0,57-3.73$ & 0.437 \\
Sphincterotomy & 1.14 & $0.66-1.97$ & 0.630 \\
Stenting & 0.62 & $0.36-1.06$ & 0.078 \\
Sphincterotomy + stent & 1.31 & $0.79-2.18$ & 0.303 \\
Urgency of the procedure & 0.79 & $0.34-1.85$ & 0.584 \\
\hline ASA Amican
\end{tabular}

ASA American Society of Anesthesiologists. ASA groups were separated into classes I-II vs. classes III-IV 
elderly. Similar to many countries worldwide, the Italian population is progressively aging, [27] and the absolute number of older people that will need ERCP procedures specifically in the future will increase. The possible implementation of a user-friendly and rapid tool for older patients' assessment will benefit both health professionals as well as their patients, reducing selection biases and improving their risk management. This will potentially and ultimately contribute to contain the incidence of post-ERCP complications and to test the real impact on the patient when the procedure is performed at an advanced age.

\section{Abbreviations}

ASA: American Society of Anesthesiologists; CBD: common bile duct:

CCl: Charlson Comorbidity index; CGA: comprehensive geriatric assessment;

DASI: Duke Activity Status Index; ERCP: Endoscopic Retrograde

Cholangiopancreatography

\section{Acknowledgements}

Dr. Pasinetti holds a career scientists award at the James J. Peters Veterans Affairs Medical Center (Bronx, NY).

\section{Funding}

The present manuscript was unfunded.

\section{Availability of data and materials}

The dataset used and analyzed during the current retrospective study are available from the corresponding author on reasonable request.

\section{Authors' contributions}

MG, PM, GB, GMP and GA designed the study. MD took care of the patients and performed endoscopy procedures. $\mathrm{MG}, \mathrm{BV}, \mathrm{MD}$ and $\mathrm{PM}$ retrieved the data and collected them in a single dataset. MG, BV and PM calculated the derived indexes. MG and PM analyzed the data. BV, GMP, PM, GB and GA interpreted the results. MG, GB and BV prepared the Tables. MG, PM and MD drafted the manuscript. GB, GMP and GA critically reviewed the manuscript. All authors read and approved the final manuscript.

\section{Ethics approval}

The study was approved by the local ethics committee of University of MilanoBicocca. All patients (or their legal representatives) signed an informed consent form to undergo the endoscopy procedure and for the use of their clinical data. Personal information has been de-identified according to the Italian legislation "Data Protection Code - Legislative Decree no. 196/2003."

\section{Consent for publication}

Not applicable.

\section{Competing interests}

The authors declare that they have no competing interests.

\section{Publisher's Note}

Springer Nature remains neutral with regard to jurisdictional claims in published maps and institutional affiliations.

\section{Author details}

'University of Milano-Bicocca, School of Medicine and Surgery, U8 Building, Floor 4, Lab 4045, Via Cadore, 48 - 20900, Monza, MB, Italy. ${ }^{2}$ NeuroMl - Milan Center for Neuroscience, Clinical Neurosciences research area, Milan, MI, Italy. ${ }^{3}$ San Gerardo Hospital ASST Monza, Acute Geriatrics Unit, Monza, MB, Italy. ${ }^{4}$ San Gerardo Hospital ASST Monza, Endoscopy Unit, Monza, MB, Italy. ${ }^{5}$ Icahn School of Medicine at Mount Sinai, New York, NY, USA. ${ }^{6}$ James J Peters Veterans Affairs Medical Center, Bronx, NY, USA.
Received: 23 August 2017 Accepted: 5 March 2018

Published online: 14 March 2018

\section{References}

1. Ukkonen M, Siiki A, Antila A, Tyrvainen T, Sand J, Laukkarinen J. Safety and efficacy of acute endoscopic retrograde cholangiopancreatography in the elderly. Dig Dis Sci. 2016;61:3302-8.

2. Ali M, Ward G, Staley D, Duerksen DR. A retrospective study of the safety and efficacy of ERCP in octogenarians. Dig Dis Sci. 2011;56:586-90.

3. Lu Y, Chen L, Jin Z, Bie LK, Gong B. Is ERCP both effective and safe for common bile duct stones removal in octogenarians? A comparative study. Aging Clin Exp Res. 2016;28:647-52.

4. Day LW, Lin L, Somsouk M. Adverse events in older patients undergoing ERCP: a systematic review and meta-analysis. Endosc Int Open. 2014;2:E28-36.

5. Holt BA. Increased severity of post-endoscopic retrograde cholangiopancreatography complications in the elderly: an issue to be addressed. Digestive endoscopy : official journal of the Japan Gastroenterological Endoscopy Society. 2014;26:534-5.

6. American Society of Anesthesiologists. New classification of physical status. Anesthesiology. 1963:24:111.

7. Ellis G, Whitehead MA, O'Neill D, Langhorne P, Robinson D. Comprehensive geriatric assessment for older adults admitted to hospital. Cochrane Database Syst Rev. 2011:CD006211.

8. Charlson ME, Pompei P, Ales KL, Mackenzie CR. A new method of classifying prognostic comorbidity in longitudinal studies: development and validation. J Chronic Dis. 1987:40:373-83.

9. Cotton PB, Lehman G, Vennes J, et al. Endoscopic sphincterotomy complications and their management: an attempt at consensus. Gastrointest Endosc. 1991;37:383-93.

10. Han SJ, Lee TH, Kang BI, et al. Efficacy and safety of therapeutic endoscopic retrograde cholangiopancreatography in the elderly over 80 years. Dig Dis Sci. 2016;61:2094-101.

11. Baillie J. ERCP in the elderly: handle with care. Endosc Int Open. 2014:2:E43-4.

12. Tohda G, Ohtani M, Dochin M. Efficacy and safety of emergency endoscopic retrograde cholangiopancreatography for acute cholangitis in the elderly. World J Gastroenterol. 2016;22:8382-8.

13. Kanamori A, Kiriyama S, Tanikawa $M$, et al. Long- and short-term outcomes of ERCP for bile duct stones in patients over 80 years old compared to younger patients: a propensity score analysis. Endosc Int Open. 2016;4:E83-90.

14. Glomsaker T, Hoff G, Kvaloy JT, et al. Patterns and predictive factors of complications after endoscopic retrograde cholangiopancreatography. $\mathrm{Br} J$ Surg. 2013;100:373-80

15. Yun DY, Han J, Oh JS, Park KW, Shin IH, Kim HG. Is endoscopic retrograde cholangiopancreatography safe in patients 90 years of age and older? Gut and liver. 2014:8:552-6.

16. Nishikawa T, Tsuyuguchi T, Sakai Y, et al. Old age is associated with increased severity of complications in endoscopic biliary stone removal. Digestive endoscopy : official journal of the Japan Gastroenterological Endoscopy Society. 2014:26:569-76.

17. Fritz E, Kirchgatterer A, Hubner D, et al. ERCP is safe and effective in patients 80 years of age and older compared with younger patients. Gastrointest Endosc. 2006:64:899-905.

18. Katsinelos P, Paroutoglou G, Kountouras J, Zavos C, Beltsis A, Tzovaras G Efficacy and safety of therapeutic ERCP in patients 90 years of age and older. Gastrointest Endosc. 2006;63:417-23.

19. Rodriguez-Gonzalez FJ, Naranjo-Rodriguez A, Mata-Tapia I, et al. ERCP in patients 90 years of age and older. Gastrointest Endosc. 2003;58:220-5.

20. Lukens FJ, Howell DA, Upender S, Sheth SG, Jafri SM. ERCP in the very elderly: outcomes among patients older than eighty. Dig Dis Sci. 2010; 55:847-51.

21. Gronroos JM, Salminen P, Laine S, Gullichsen R. Feasibility of ERCP procedures in patients 90 years of age and older. J Clin Gastroenterol. 2010;44:227-8.

22. Sugiyama M, Atomi Y. Endoscopic sphincterotomy for bile duct stones in patients 90 years of age and older. Gastrointest Endosc. 2000;52:187-91.

23. Finkelmeier $F$, Tal A, Ajouaou M, et al. ERCP in elderly patients: increased risk of sedation adverse events but low frequency of post-ERCP pancreatitis. Gastrointest Endosc. 2015;82:1051-9.

24. Garcia CJ, Lopez OA, Islam S, et al. Endoscopic retrograde cholangiopancreatography in the elderly. Am J Med Sci. 2016;351:84-90. 
25. Committee ASOP. Anderson MA, fisher $L$, et al. complications of ERCP. Gastrointest Endosc. 2012;75:467-73.

26. Park TY, Choi JS, Oh HC, Kim JW, Do JH, Jung YH. Assessment of safety of non-anesthesiologist-assisted endoscopic retrograde

cholangiopancreatography based on performance status in elderly patients. J Gastroenterol Hepatol. 2014;29:1943-8.

27. Mazzola P, Rimoldi SM, Rossi P, et al. Aging in Italy: the need for new welfare strategies in an old country. Gerontologist. 2016 Jun;56(3):383-90.

Submit your next manuscript to BioMed Central and we will help you at every step:

- We accept pre-submission inquiries

- Our selector tool helps you to find the most relevant journal

- We provide round the clock customer support

- Convenient online submission

- Thorough peer review

- Inclusion in PubMed and all major indexing services

- Maximum visibility for your research

Submit your manuscript at www.biomedcentral.com/submit 